\title{
INFECÇÕES NATURAIS DE MAMIFEROS SILVESTRES EM ÁREA ENDEMICA DE LEISHMANIOSE TEGUMENTAR DO ESTADO DE SÃO PAULO, BRASIL *
}

\section{RSPSP-138}

Foratrini; O. P. et al. - Infecções natu. rais de mamiferos silvestres em área endêmica de leishmaniose tegumentar do Estado de São Paulo, Brasil. Rev. Saúde públ., S. Paulo, 6: 255-61, 1972.

Resumo - Descreve-se a presença de foco de leishmaniose tegumentar no vale do rio Moji-Guacú, em regiấo comum aos municipios de Luiz Antonio, São Carlos, Rincão e Santa Rita do Passa Quatro, no Estacio de São Paulo, Brasil. Os casos humanos apresentavam formas clínicas caracterizadas por lesões úlcerovegetantes, de evolução lenta e pobres em parasitos. As investigaçôes sobre infecção natural em animais silvestres levou ao iso. lamento de roedores, de três cepas em cultura, duas procedentes de Akodon arviculoides e uma de Oryzomys nigripes. As provas de inoculação em hamsters foram, até o momento, positivas para duas delas, mas com evolução lenta, com manifestações clinicas muito discretas e pobres em parasitos. Pelos dados disponiveis ate o momento, parece tratar-se de cepas filiáveis à raça "lenta", à qual se atribui papel na etiologia da forma cutaneo-mucosa da leishmaniose.

\author{
Oswaldo Paulo FORAT'TINI ** \\ Dino B. G. PATTOLI ** \\ Ernesto Xavier RABELLO ** \\ Octávio Alves FERREIRA **:*
}

\begin{abstract}
UNITERMOS - Leishmaniose tegumentar (reservatórios)*; Akodon arviculoides (infecção leishmaniótica natural)*; Oryzomys nigripes (infecção leishmaniótica natural)"; Endemia leishmaniotica; $E n$ zootia leshmaniótica; Raça "lenta" de leishmaniose tegumentar.
\end{abstract}

\section{NTROD U G A O}

Em nota anterior, noticiou-se a existência de área endêmica de leishmaniose tegumentar, situada na região nordeste do Estado de São Paulo, Brasil (Forstrinr et al. 2 , 1972). Nessa oportunidade, foram detectados quatro casos humanos, cujas manifestações clínicas revelaram-se através lesões de aspecto úlcero.vegetantes, de evolução lenta e pobres em formas parasitária. Tais achados ensejaram a programação de série de investigações destinadas a elucidar aspectos epidemiológicos ainda mal conhecidos. Visaram elas pesquisar a existência de focos naturais e o mecanismo de transmissão local da

* Trabalho realizado com o auxílio parcial da Fundação de Amparo à Pesquisa do Estado de São Paulo (Proc. C. Médicas 70/788).

* Do Departamento de Epidemiologia da Faculdade de Saúde Pública da USP - Av. Dr. Ar. naldo, 715 - Sảo Paulo, SP, Brasil.

**:* Da Diretoria de Combate a Vetores da Superintendência do Saneamento Ambiental (SUSAM) do Estado de São Paulo - Rua Tamandaré, 649 - São Paulo, SP, Brasil. 
FORATTINI, O. P. et al. - Infecçóes naturais de mamíferos silvestres em área endêmica de leishmaniose tegumentar do Estado de São Paulo, Brasil. Rev. Saúde públ., S. Paulo, 6: 255.61. 1972.

parasitose. Os primeiros resultados obtidos são relatados neste trabalho e referem-se a encontros de infecçōes naturais em mamíferos silvestres.

\section{Região estudada}

A área é formada por partes dos municípios de Luis Antonio, São Carlos, Rincão e Santa Rita do Passa Quatro, situada aproximadamente a $21^{\circ} 50^{\prime}$ de latitude sul e $48^{\circ}$ de longitude oeste e próxima das cidades de Ribeirão Preto, Araraquara e São Carlos (Figura 1).

A região faz parte do vale do rio MojiGuaçú, que pertence ao sistema hidrográfico do Rio Grande. A altitude média local oscila ao redor dos $500 \mathrm{~m}$ acima do nível do mar. $O$ relevo é ligeiramente on. dulado, constituído por numerosos vales que acompanham pequenos cursos de água, afluentes daquele rio. A vegetação primitiva é relativamente escassa, representada por algumas glebas de mata que acompanham as margens do Moji-Guaçú e por cerrados que se estendem mais em direção aos espigões. As terras sofreram intensamente as consequiências da atividade humana, daí resultando campos de cultura ou vegetações de segunda formação.

As pesquisas foram levadas a efeito em área de floresta primitiva, conhecida como Mata do Beija-Flor e pertencente à Fazenda Jataí, onde funciona a Estação Experimental de Luiz Antonio, da Secretaria da Agricultura. Na margem oposta, . estende-se o Bairro Falcão que também foi escolhido como sede de investigações e onde foram assinalados vários casos humanos da doença. Outros dados foram observados nas Fazendas União e Vaçununga. Nesta última, observa-se o terreno devastado em grande extensão, restando apenas matas de segunda formação confinadas ao fundo dos vales e onde foram encontrados alguns doentes. As Fi- guras 2 a 5 dão idéia dos aspectos supradescritos.

Até o momento, com a inclusão daque. les já descritos anteriormente (ForatiIn et al. 2,1972 ), o número total de casos humanos ascende a nove, assim distribufdos:

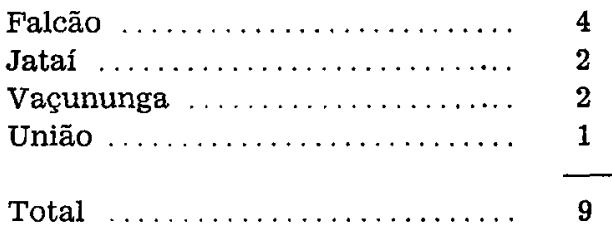

Todos mostraram aspecto clínico bastante uniforme, com lesões precocemen. te vegetantes e de evolução longa. Em um deles, observou-se comprometimento mucoso nasofaringeano.

\section{MATERIAL E METODOS}

A pesquisa de infecção natural em animais foi feita mediante a captura e o transporte dos exemplares vivos ao laboratório. Uma vez ali, eram submetidos ao exame do tegumento para verificar a possível presença de lesōes macroscópicas. Tanto na presença como na ausência dessas alterações cutâneas, procediase à coleta de material para cultura, de acordo com a técnica descrita por HerRer et al. 3 (1966). Rotineiramente, foi obedecida a seguinte sequiência:

1 - retirava-se fragmentos cutâneos em vários pontos da cauda, preferivelmente ao longo da metade basal, e da extremidade dos pavilhões auriculares. No caso da presença de lesões macroscópicas, eram elas preferentemente incluídas na colheita;

2 - precedendo a retirada, a região era desinfetada com álcool iodado a $7,0 \%(7,0 \%$ de iodo e $3,0 \%$ de iode. to de potássio). Antes da secagem, era feita limpeza, a mais completa possível, com éter sulfúrico; 
FORATTINI, O. P. et al. - Infecções naturais đe mamiferos silvestres em área endêmica de leishmaniose tegumentar do Estado de São Paulo, Brasil. Rev. Saúde públ., S. Paulo, 6: 255_61. 1972.

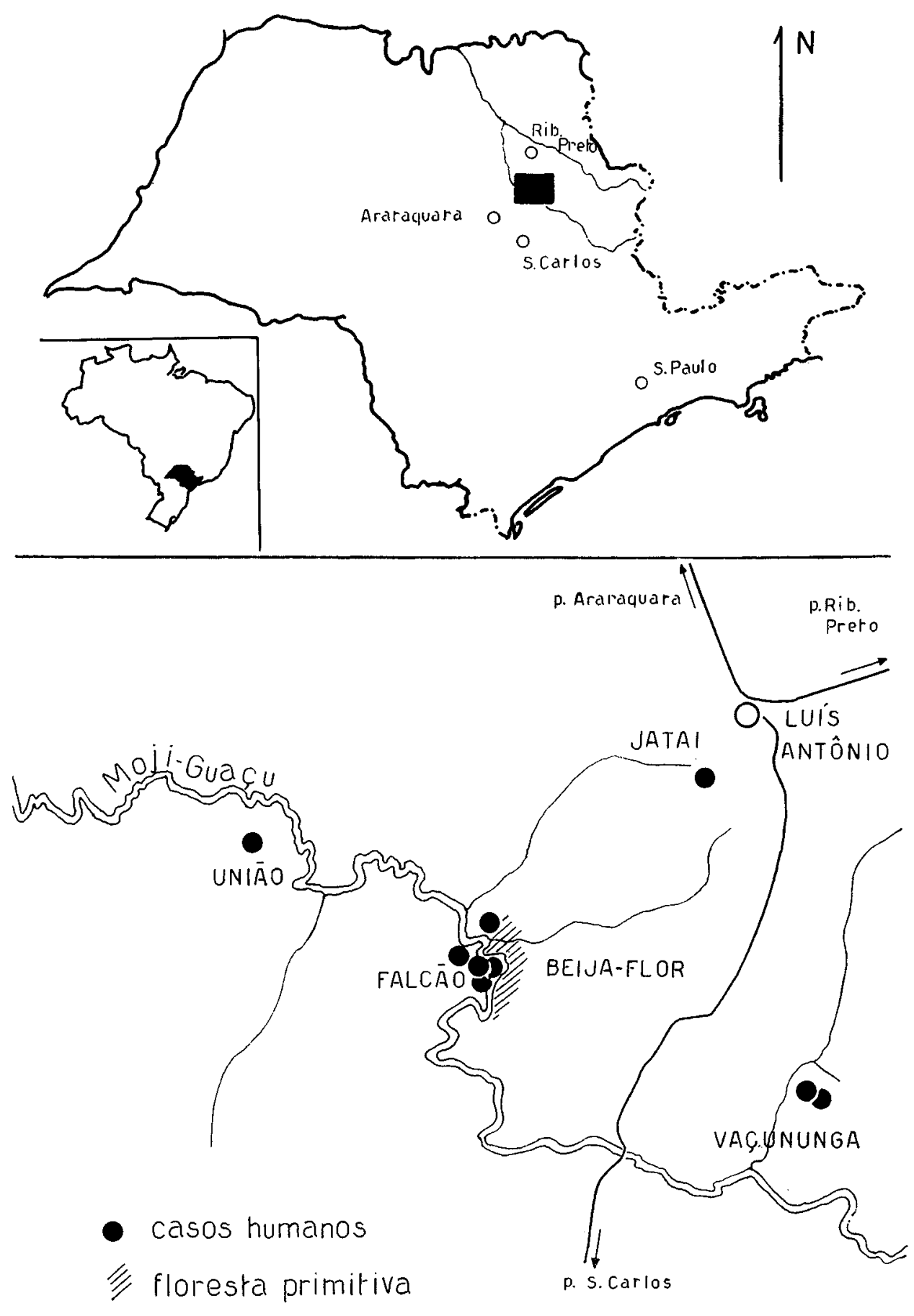

Fig. 1 - Localização da área estudada, mostrando a distribuição dos casos humanos observados 
FORATTINI, $O . P$. et al. - Infecçóes naturais de mamiferos silvestres em área endêmica de leishmaniose tegumentar do Estado de São Paulo, Brasil. Rev. Saúde públ., S. Paulo, 6: 255.61 1972 .

3 - o material retirado era cortado em fragmentos finos, com o auxílio de tesoura e colocado em solução salina, contendo penicilina e estreptomicina, na seguinte concentração:

Penicilina G potássica $\cdot 1.000 \mathrm{U}$ por $\mathrm{ml}$

Sulfato de estreptomicina $1 \mathrm{mg}$ por $\mathrm{ml}$

4 - os frascos com os fragmentos cu. tâneos eram mantidos em refrigerador a $4^{\circ} \mathrm{C}$, durante $\mathbf{7 2}$ horas;

5 - após retirada da solução salina, os fragmentos eram triturados em geral e suspensos em $2 \mathrm{ml}$ de solução salina com penicilina e estreptomicina nas mesmas concentrações já citadas.

O meio de NNN foi utilizado não apenas para $O$ isolamento, mas também para a manutenção de culturas em repiques sucessivos.

As cepas isoladas foram inoculadas por via intradérmica, na região nasal de hamsters. Estes animais foram submetidos ao exame periódico para detectar a ocorrência de alterações cutâneas. Após tempo variável de $2 / 3$ meses, com ou sem desenvolvimento de lesões, procediase à retirada da regiāo inoculada para exame histológico.

RES ULTA DOS

Infecção natural - Na Mata do BeijaFlor, foram coletados e examinados 137 roedores, cujos resultados encontram-se na Tabela 1:

T A B E L A

Coleta e exame de roedores, na mata de Beija-Flor

\begin{tabular}{l|r|r}
\hline \multicolumn{1}{c|}{ Espécie } & $\mathrm{N} \cdot{ }^{\circ}$ & Culturas positivas \\
\hline \hline Akodon arviculoides & 50 & 2 \\
Clyomys laticeps & 2 & - \\
Clyomys sp. & 1 & - \\
Nectomys squamipes & 2 & - \\
Nectomys sp. & 2 & - \\
Oryzomys nigripes & 30 & - \\
Oryzomys subflavus & 33 & 3 \\
Oryzomys sp. & 4 & - \\
Zygodcntomys sp. & 137 & \\
\hline Total & & \\
\hline
\end{tabular}

Como se pode ver, foi conseguido o isolamento de 3 cepas, 2 procedentes de Akodon arviculoides e uma de Oryzomys nigripes. $E$ de se assinalar que houve frequiente contaminação por cogumelos a qual, em alguns casos, ultrapassou $\mathbf{5 0} \%$ das semeaduras. Nos três isolamentos, somente um dos animais, representado por exemplar de Akodon arviculoides, apresentava lesões macroscópicas, limitadas à cauda e com aspecto de pequenas áreas escamativas próximas à base (Figura 6). Tais alterações eram pequenas, com apenas alguns milímetros de largura, um tanto esparsas e cobertas de escamas que lhes emprestava tonalidade es. branquiçada. 
FORATTINI, O. P. et al. - Infecções naturais de mamíferos silvestres em área endêmica de leishmaniose tegumentar do Estado de São Paulo, Brasil. Rev. Saúde públ., S. Paulo, 6: 1972 .

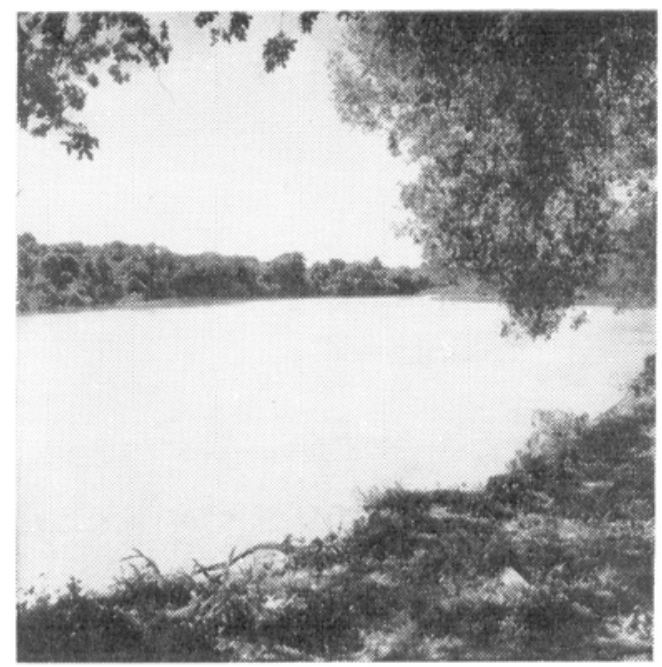

Fig. 2 - Aspecto do rio Moji-Guacú mostrando trecho da Mata do Beija_Flor, na margem oposta.

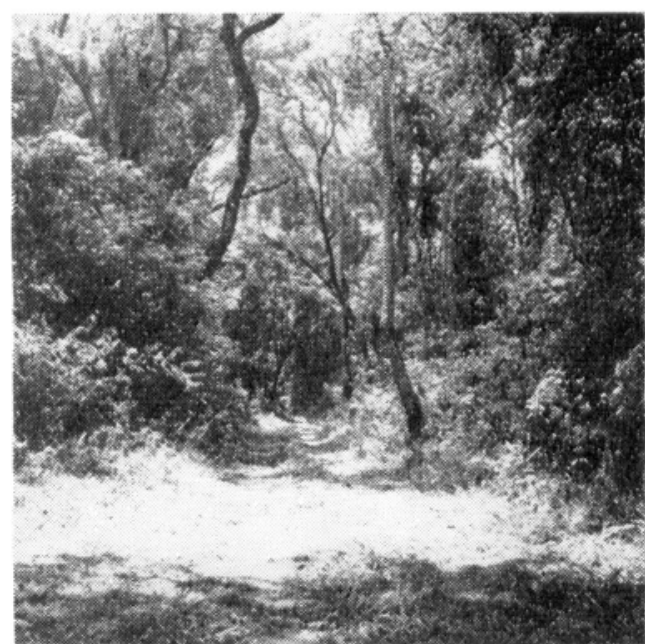

Fig. 3 - Trecho da Mata do Beija-Flor, de aspecto primitivo.

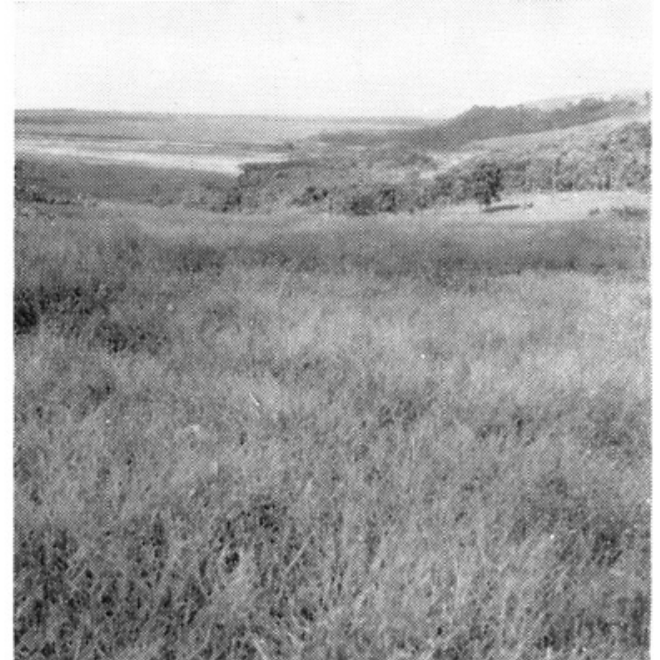

Fig. 4 - Aspecto panoramico da Fazenda Va. çununga, notando-se florestas secundárias confinadas no fundo dos vales. 
FORATTINI, O. P. et al. - Infecções naturais de mamíferos silvestres em área endêmica de leishmaniose tegumentar do Estado de São Paulo, Brasil. Rev. Saúde públ., S. Paulo, 6: 1972 .

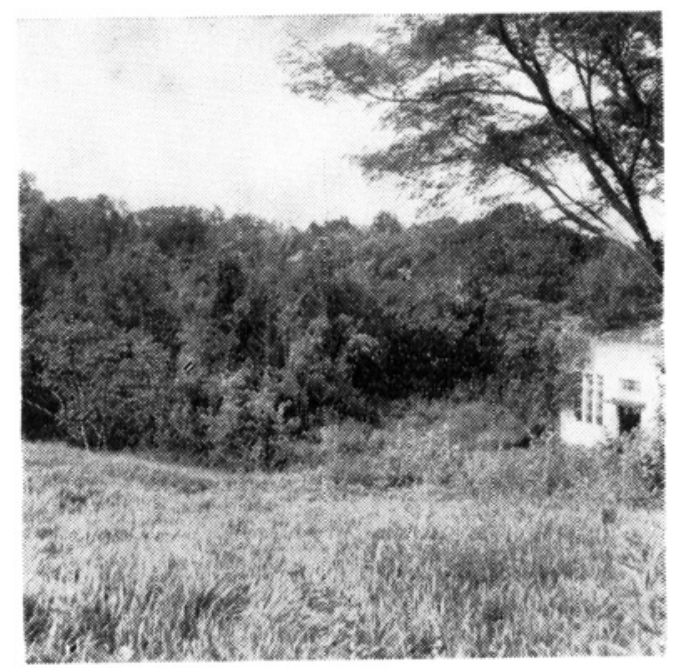

Fig. 5 - Mata secundária de vale na Fazenda Vaçununga.

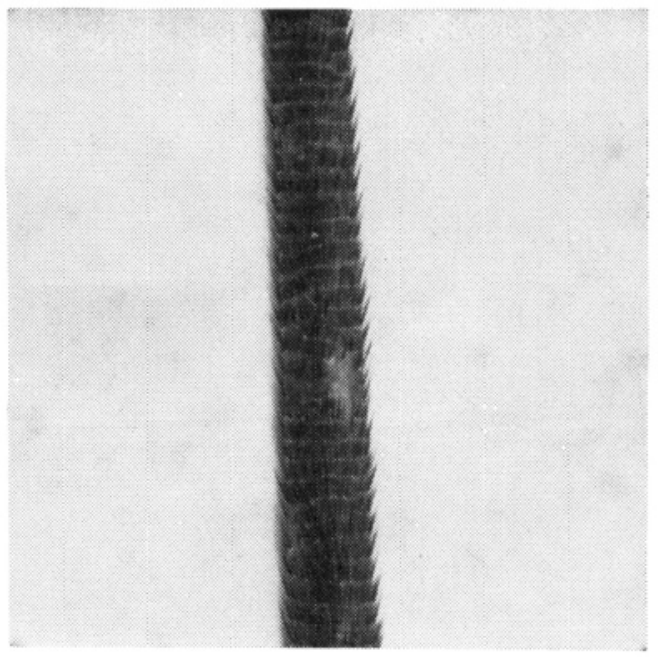

Fig. 6 - Lesão escamativa na cauda de Akodon arviculoides.

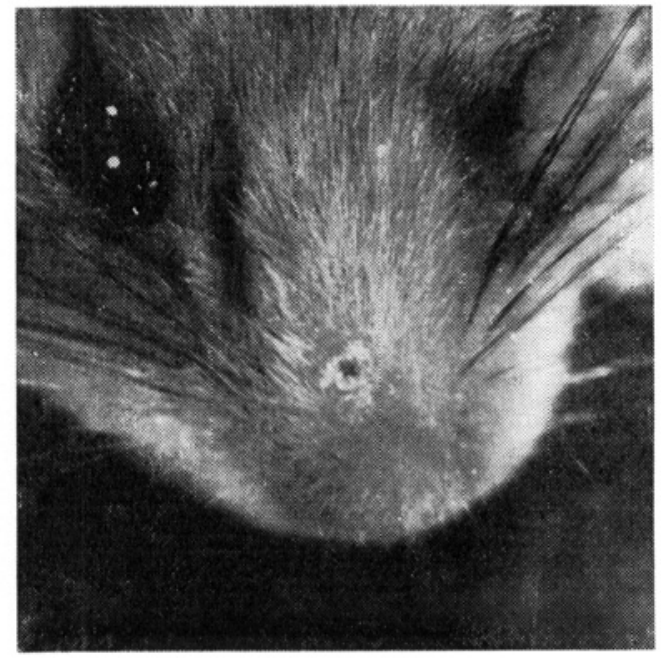

Fig. 7 - Lesão nodular crostosa obtida em hamster com a eepa $R$, após 40 dias de evolu. cão, positiva para formas de leishmania. 
FORATTINI, O. P. et al. - Infecções naturais de mamíferos silvestres em área endêmica de leishmaniose tegumentar do Estado de Săo Paulo, Brasil. Rev. Saúle públ., S, Paulo, 6: 255_61. 1972 .

Identificação - A natureza leishmaniótica dos isolamentos foi comprovada através inoculaçōes em laboratório, leva. das a efeito em hamsters.

As cepas foram designadas pelas siglas seguintes:

$\mathbf{R}_{1}(\operatorname{RPX} 1965)-$ Akodon arviculoides

$\mathrm{R}_{2}$ (RPX 1688) - Akodon arviculoides

$\mathrm{R}_{3}$ (RPX 1703) - Oryzomys nigripes

Até o momento, foram obtidos os seguintes resultados:

1. ${ }^{a}$ Inoculação - Em 8.II.72, primeiro repique das culturas em hamsters jovens.

$R_{1}-$ Em 17.IIr.72, observou-se a presença de ligeiros nódulos crostosos na região inoculada (Figura 7). Em 9.VI.72, $o$ aspecto mostrava-se inalterado. Um dos animais foi sacrificado e o exame histológico revelou-se positivo para formas em leishmania.

As outras duas cepas apresentaram resultados negativos até o momento.

2. ${ }^{a}$ Inoculação - Em 22.II.72, terceiro repique das culturas em hamsters jovens. Nada pode-se observar até agora. Os animais continuam em observação.

3." Inoculação - Em 24.III.72, quarto repique das culturas, em dois lotes de hamsters jovens para cada cepa. Um deles normal e outro tratado com corticoides. Em 1.IV.72 e 4.IV.72, morreram dois animais dos lotes $R_{3}$ e $R_{1}$, respectivamente, e que tinham sido submetidos à ministração da droga. O exame histológico da região inoculada revelou a pre. sença đe formas parasitárias em leishmânia. Os outros animais continuam em observação, até o momento.

Assim sendo, até agora somente se revelaram positivas as cepas $R_{1}$ e $R_{3}$ e, apenas ao exame histológico de animal tratado. Os demais continuam em obser. vação.
$E$ de se notar que houve frequiente desenvolvimento de nódulos, peladas e crostas na região inoculada sem, contudo, ainda se revelarem positivas à pesquisa de parasitos. Dessa maneira, a identificação completa das culturas isoladas está sendo tentada, esperando-se obter maiores informaçōes em data posterior.

COMENTARIOS

Em épocas mais recentes, tem-se acumulado o número de dados sobre encontro de infecções leishmanióticas naturais em animais silvestres da região neotropical. $\mathbf{E}$ isso, graças a investigações levadas a efeito em diversas áreas centro e sulamericanas. No caso particular do Brasil, as investigações de Lainson \& Shaw 4 (1970) revelaram certas feiçōes a serem utilizadas na interpretação dos achados dessa natureza. As pesquisas desses autores na região norte do Estado de Mato Grosso, levaram à diferenciação de duas raças do parasita, denominadas "rápida" (fast) e "lenta" (slow). As duas podem coexistir na mesma área, diferen. ciando-se pelo comportamento em inoculações experimentais e em cultura. A primeira tende a produzir lesões em pou$\cos$ meses, com produção de grande número de histiocitos e formas em leishmânia. A segunda é de evolução bem mais vagarosa, com a produção de lesões discretas, nodulares, dotadas de reação tissular evidente, mas pobres em parasitos.

Pelos dados obtidos até agora, tudo leva a crer que as cepas isoladas por nós devam ser classificadas como da forma "lenta", pois as lesōes foram discretas, pouco produtivas e pobres em leishmânias. Nas citadas investigações executadas em Mato Grosso, a espécie encontrada naturalmente infectada por essa raç "lenta" foi a correspondente ao roedor Oryzomys concolor. A ela poderíamos acrescentar, com estas observações no Estado de São Paulo, possível papel aná. 
FORATTINI, $O$. $P$. et al. - Infeccōes naturais de mamíferos silvestres em área endêmica de leishmaniose tegumentar do Estado de São Paulo, Brasil. Rev. Saúde públ., S. Paulo, 6: 255_61. 1972 .

logo desempenhado por Akodon arviculoides $e$ Oryzomys nigripes. Este último, aliás, já tendo sido observado como pa. rasitose natural por formas em leishmânia no Estado do Rio de Janeiro (BARBOSA et al. 1,1970 ).

Em principio, a cepa "lenta" seria filiável à etiologia da forma cutâneo-mucosa da doença. Nos casos humanos observados na região, pudemos verificar o desenvolvimento de lesões mucosas em um deles mas, como regra geral, todos eram portadores de pequeno número de lesões, pobres em parasitos e de aspecto produtivo. Assim sendo, quer-nos parecer que, pelo menos, o comportamento das cepas $R_{1}$ e $R_{3}$ é de molde a levar-nos à hipótese de certa identidade com os casos humanos locais. As provas de inoculação das cepas humanas encontram-se em andamento e serão comunicadas oportunamente.

Por outro lado, a elevada contaminação que ocorreu nas tentativas de isola. mento, impede a estimativa de coeficientes de infecção natural. Para obviar esse inconveniente, nas observações em continuação introduziu-se, de maneira rotineira, o exame histológico do material retirado.

De qualquer forma, estas observações acrescentam às já realizadas em outras partes do Brasil e pela primeira vez, a possível responsabilidade epidemiológica de Akodon arviculoides e Oryzomys nigripes como reservatórios da leishmaniose tegumentar no Estado de São Paulo.

\section{O N CLUSOES}

Face ao apresentado, é lícito concluir:

1 - Existe região endêmica de leishmaniose tegumentar no vale do rio MojiGuaçú, na altura dos municípios de Luis Antonio, São Carlos, Rincão e Santa Rita do Passa Quatro, no Estado de São Pau. lo, Brasil.

2 - Essa endemia está provavelmente filiada a foco enzoótico, do qual participam roedores. Entre estes, provaram encontrar-se como portadiores da infecção natural, representantes de Akodon arviculoides e Oryzomys nigripes.

3 - As cepas que infectam esses animais, pelo menos duas delas, parecem pertencer à raça "lenta" de Lainson \& Shaw 4 (1970) que, por sua vez, encontra-se associada à etiologia da forma cutâneo-mucosa da parasitose.

4 - Tudo leva a crer que as cepas isolajas desses animais têm comportamento semelhante às de origem humana dos casos encontrados na região.

A GRA DECIMENTO

Ao Dr. Cory T. de Carvalho, sob cuja supervisão procedeu-se à identificação dos mamíferos coletados.

RSPSP-138

Forattini, O. P. et al. [Natural infection of sylvatic mammals of cutaneous leishmaniasis in S. Paulo State, Brazil.] Rev. Saúde públ., S. Paulo, 6: 255.61, 1972.

SumMary - An endemic area of cuta neous leishmaniasis in the State of $\mathbf{S}$. Paulo, Brazil, is described. Clinical aspects of human cases showed few lesions with slow evolution and few parasites. From investigations on natural infections of local sylvatic mammals by skin cultures technique ensued three strains isolations from Akodon arviculoides and Oryzomys nigripes rodents. Two of these showed to infect hamsters experimentally, but with discrete cutaneous manifestations and few parasites. This aspect seems to be alike the behaviour of "slow" strain, who was included in the possibility of the mucocutaneous leishmaniasis etiology. The pre- 
FORATTINI, O. P. et al. - Infecçóes naturais de mamiferos silvestres em área endêmica de leishmaniose tegumentar do Estado de São Paulo, Brasil. Rev. Saúde públ., S. Paulo, 6: 255.61. 1972 .

sence of sylvatic enzootic forms of the disease is concluded.

UNITERMS - Cutaneous leishmaniasis reservoirs"; Akdon arviculoides, natural leishmanial infection*; Oryzomys nigripes, natural leishmanial infection*; Mucocutaneous leishmaniasis; Sylvatic enzootic forms of cutaneous leishmaniasis; Endemic area of cutaneous leishmaniasis; "Slow" strain in mucocutaneous leishmaniasis etiology.

\section{REFERENCIAS BIBLIOGRAFICAS}

1. BARBosA, F. S. et al. - Nota sobre a infecção natural de roedores por Leishmania sp. nos limites dos municipios de Teresópolis - Nova Friburgo, Estado do Rio de Janeiro. Rev. Soc. bras. Med. trop., 4: 113-5, 1970.
2. FORATTINI, O. P. et al. Nota sobre um foco de leishmaniose tegumentar na região nordeste do Estado de são Paulo, Brasil. Rev. Saúde públ., s. Paulo, 6: 103-5, 1972.

3. HERRER, A. et al. - Natural infections of Leishmania and trypanosomes de. monstrated by skin culture. J. Parasit., 52: 954-7, 1966.

4. LAINSON, R. \& SHAW, J. J. - Leishmaniasis in Brazil: $V$ - Studies on the epidemiology of cutaneous leishmaniasis in Mato Grosso State, and observations on two distinct strains of Leishmania isolated from man and forest animals. Trans. roy. Soc. trop. Med. Hyg., 64: $654.67,1970$.

Recebido para publicąão em 12.7-1972 $A_{i}$ rovado para publicasão em 26.7-1972 